\title{
CHAOTIC INFLATION AND THE OMEGA PROBLEM
}

\author{
G. J. Mathews, F. Graziani, and \\ H. Kurki-Suonio \\ Lawrence Iivermore National Laboratory \\ University of California \\ Livermore, California 94550 \\ This paper was an invited talk \\ prepared for submittal to the \\ Workshop on Primordial Nucleosynthesis \\ and Evoiution of the Early Universe \\ Tokyo, Japan \\ September 3-8, 1990
}

December, 1990

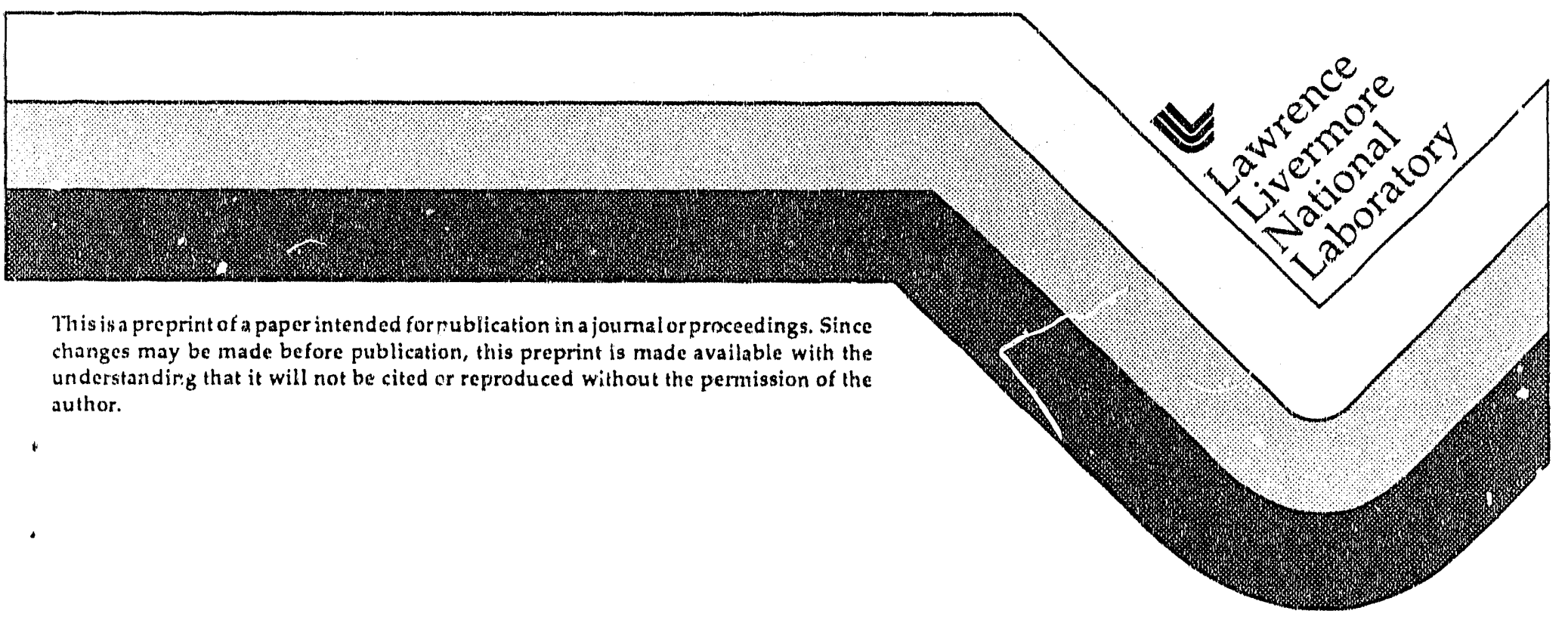


DISCI.AIMER

This document was preparted as an uccount of work sponsored by an agency of the Inited stutes Government. Neither the United States Government nor the Liniversity of Californit nor uny of their emplnyees, mabes uny watranty, express or implied, or assumes an! leul liuhility of responsibility for the accuracy, conpleteness, or usefulne'ss of any information. apparalus. product, of process disclosied, or represents that its use would not infringe privately ouned rights. Ruference herein to any specific commercial products. process, or service by trade name, Irademark. manufacturer. or othermise. does not necessarily constitute of imply its endorsement, recommendation, of favoring hy the United Siates Government or the University of California. The viens and opinions of authors expressed herein do not necessarily state or reflect thowe of the Inited States Government or the University of California, and shall not be used for advertising or product endorsement purposses. 
ABSTRACT. An overview of the omega problem is presented along with discussions of several ways to resolve this problem in the context of chaotic and stochastic inflation.

\section{Introduction}

One of the most compelling features of inflationary cosmology is that it provides a solution to the flatness problem, i.e. an explanation of the fact that the ratio, $\Omega$, of the present mass density to the closure density is so close to unity. Indeed, inflationary models naturally lead to a present value of the closure parameter which is equal to unity to many significant figures. At the same time, however, there are at least some observational indications that the present value of the closure parameter may be significantly less than unity. At the very least it is probably safe to say that there is no convincing evidence that the present mass density of the universe is not less than half of the closure density. This is the omega problem. The purpose of this paper will be to take the possibility that there is an omega problem seriously and look within the context of inflationary scenarios for a natural explanation as to why the present value of the closure parameter might be so small.

\section{The Omega Problem}

Let us begin with a brief review of observational determinations of $\Omega$. There are seven methods. Three of them seem to indicate that $\Omega$ is less than unity and the others are inconclusive. The most direct determination of the closure parameter is from mass-tolight ratios. These ratios increase with distance scale from the solar neighborhood (where $M / L \sim 2$ implies $\Omega \sim 0.002$ ) to the supercluster scale (where, for example, virgocentric infall of the Local Group implies (Davis and Peebles 1983) $M / L=350, \Omega=0.35 \pm .02$ ).

The largest distance scale for which one may determine the closure pararneter is from the ratio of the streaming velocity to the Hubble velocity, $\frac{v}{v_{H}}$, of the local supercluster with respect to the cosmic microwave background. The streaming velocity is related to the closure parameter and the local density distribution via the relation (Peebles 1976),

$$
\frac{v}{v_{H}}=\Omega^{0.6} \frac{\delta \rho}{\rho}
$$

If the IRAS survey of galaxies is used to infer the mass distribution, $\frac{\delta \rho}{\rho}$, then a value of $\Omega$ near unity results (Yahil, Walker, and Rowan-Robinson 1986). However, there is reason to believe (Fukugita 1990) that the IRAS survey is biased against the large mass concentrations associated with giant ellipticals. Hence, $\frac{\delta \rho}{\rho}$ is underestimated and $\Omega$ is 
overestimated. Correcting for this bias reduces the upper limit from streaming motion to $\Omega \lesssim 0.4$.

Similarly, galaxy number counts as a function of redshift at first seemed to indicate a large value for $\Omega$ (Loh and Spillar 1986). However, it has been pointed out that there are large corrections to the luminousity function for faint galaxies (Caditz and Petrozian 1989) as well as corrections for galactic evolution and merging (Bahcall and Tremain 1988). These corrections introduce large uncertainties $(\Delta \Omega \sim \pm 1)$ and can reduce the inferred value of the closure parameter to $\Omega \sim 0.2 \pm 1.0$. On the other hand, it has recently been shown (Fukugita, et al. 1990) that galaxy number counts as a function of magnitude are only consistent with $\Omega \sim 0.1$ independent of galactic evolution corrections. Redshiftmagnitude (Yoshii and Takahara 1988) or redshift-angular-diameter studies (Sandage 1988) are presently too uncertain $(\Omega \sim 1 \pm 1)$ to add any further significant constraint.

Perhaps, it is also worth pointing out that, as we have heard at this conference (Audouze 1990; Steigmann 1990), the standard big-bang upper limit to the baryonic contribution to the closure parameter based upon primordial nucleosynthesis is $\Omega_{b} \leq 0.06$. We have also heard (Sato 1990; Mathews, et al. 1990) that an inhomogeneous model with optimum baryon diffusion could increase this limit to $\Omega_{b} \lesssim 0.3$ for $Y_{p} \lesssim 0.24$. Although it is popular to propose dark-matter candidates to increase the total $\Omega$ abcive the baryonic contribution, none of these candicates have yet been detected. It is at least possible that there is only baryonic matter and $\Omega=\Omega_{b} \lesssim 0.3$.

Hence, at the present time there is no convincing observational evidence that the universe is closed. At the very least, one can say that a value of $\Omega \lesssim 0.5$ is not presently ruled out. It is worthwhile, therefore, to consider how inflationary scenarios might be naturally altered to allow for a present closure parameter less than unity.

The present value of the closure parameter is determined from whatever curvature, $k$, may have been present prior to inflation, and the amount of expansion, i.e.

$$
\Omega=1+\frac{k}{H_{0}^{2} R_{0}^{2}},
$$

where $H_{0}$ is the present Hubble parameter, and $R_{0}$ is the present scale factor. For the purposes of this discussion we take $\frac{k}{H_{b}^{2} R_{b}^{2}} \sim-1$ just before inflation. The present value of $\Omega$ is then determined from the value of $H_{f}^{2} R_{f}^{2}$ at the end of inflation times the factors by which these parameters have changed from the end of inflation through the present matter dominated epoch. A value of $\Omega \lesssim 0.5$ corresponds to $\ln \left(\frac{R_{f}}{R_{b}}\right) \lesssim 60+\ln \left(\frac{T_{b}}{10^{15} \mathrm{GeV}}\right)$.

It should also be pointed out that there is another implicit assumption in any inflationary model which attempts to allow for a present value of the closure parameter less than unity. At the time when $\Omega$ begins to deviate from unity the observable horizon corresponds to the horizon before inflation. Fluctuations in the initial curvature would then lead to fluciuations in the present microwave background temperature. We must therefore assume that, although the initial curvature may have been large, it was smooth. We suggest at least one reason why this might be a reasonable assumption. A smooth curvature would minimize the Einstein-Hilbert action. Thus, if the birth of the universe from the Planck time can be described by a functional integral in Euclidean space-time, deviations from the minimum action would have been exponentially suppressed. 


\section{New Inflation}

It is worthwhile to first review the recent demonstration by Steinhardt (1990) that the new inflationary model (Linde 1983) can not simultaneously satisfy $\Omega \lessgtr 0.5$ and the constraint from the isotropy of the microwave background temperature, $\frac{\Delta T}{T} \lesssim 10^{-4}$. For a new inflationary potential of the form, $V(\phi)=V_{0}-\lambda \phi^{n}$, in the slow rolling approximation, the number of expansion e-folds during inflation is given by;

$$
\ln \left(\frac{R_{f}}{R_{b}}\right)=\int H d t=\frac{3 H^{2}}{\left|V^{\prime \prime}\right|}=\frac{3 H^{2}}{\lambda n(n-1) b_{b}^{n-2}}
$$

whereas, the background fluctuations are given by (Guth and $\mathrm{Pi}$ 1986; Steinhart and Turner 1986),

$$
\frac{\Delta \rho}{\rho}=\frac{4 \Delta T}{T}=\frac{H^{2}}{\dot{\phi}_{h}}
$$

where $\dot{\phi}_{h}$ is evaluated during the inflationary epoch at the point when the present horizon stretches beyond the apparent horizon, $H^{-1}$. For an inflation occuring at $T \sim 10^{15} \mathrm{GeV}$, this occurs about 60 e-folds before the end of inflation (cf. Sec. 2). Thus,

$$
60 \approx \frac{3 H^{2}}{\lambda n(n-1) \phi_{h}^{n-2}}
$$

In the slow rolling approximation,

$$
\dot{\phi}_{h} \approx \frac{-V^{\prime}\left(\phi_{h}\right)}{3 H}=\frac{\lambda n \phi_{h}^{n-1}}{3 H} .
$$

Thus,

$$
\frac{\Delta \rho}{\rho}=\left(\frac{3 H^{2}}{\lambda n(n-1) \phi_{h}^{n-2}}\right)\left(\frac{(n-1) H}{\phi_{h}}\right) .
$$

This implies that $\phi_{h} \gtrsim 6 \times 10^{5}(n-1) H$. Taking the ratio of eq. (3.1) to (3.3) and setting $\phi_{b} \approx H$ implies,

$$
\frac{\ln \left(R_{f} / R_{b}\right)}{60} \gtrsim\left[6 \times 10^{5}(n-1)\right]^{n-2} \text {. }
$$

For any potential with $n>2$ then one requires $\ln \left(R_{f} / R_{b}\right) \gg 100$ and a present closure parameter equal to unity to many significant figures.

\section{Chaotic Inflation}

The constraint derived above does not, however, apply to chaotic inflationary scenarios (Linde 1983). Such models are characterized by the presence of domains of homogeneous inflaton field which appear shortly after the Planck time. For a simple $V(\phi)=\lambda \phi^{n}$ potential the constraint conditions can be derived as above except that now one can have $\phi_{b}>\phi_{h}>H$.

Figure 1 shows numerical caiculations of the present closure parameter and microwave background energy-density fluctuations as a function of the initial value for the inflaton 
field, $\phi_{0}$, for different values of the potential strength, $\lambda$, for a $V(\phi)=\frac{\lambda}{4} \phi^{4}$ potential. It can be seen that $0.1 \lessgtr \Omega \lesssim 0.5$ implies an initial inflaton field, $\phi_{0} \approx 4.5 \pm 0.1$, depending upon $\lambda$. Models with smaller $\lambda$ require somewhat smaller $\phi_{0}$. When $\lambda$ is small it takes a longer time before the vacuum energy density exceeds the particle energy density. Thus, the inflation happens later, with a longer time scale, leading to a lower reheating temperature. After inflation the universe needs to expand by a smaller factor to reach the present temperature. Correspondingly, to have $\Omega$ deviate from unity by the present time requires less inflation. At the same time a value of $\lambda \lesssim 10^{-12}$ gives $\frac{\Delta \rho}{\rho} \leq 10^{-4}$. For small enough $\phi_{0}$ that $\Omega$ presently deviates significantly from unity, this is satisfied with a somewhat smaller $\lambda$. (for $\Omega=0.5, \lambda \leq 10^{-13}$; for $\Omega=0.1, \lambda \leq 10^{-14}$ ). This is because fluctuations with a wavelength on the scale of the present microwave background surface of last scattering were in this case generated at the very beginning of inflation, when $\dot{\phi}$ had not yet accelerated to its "slow-rolling" value.

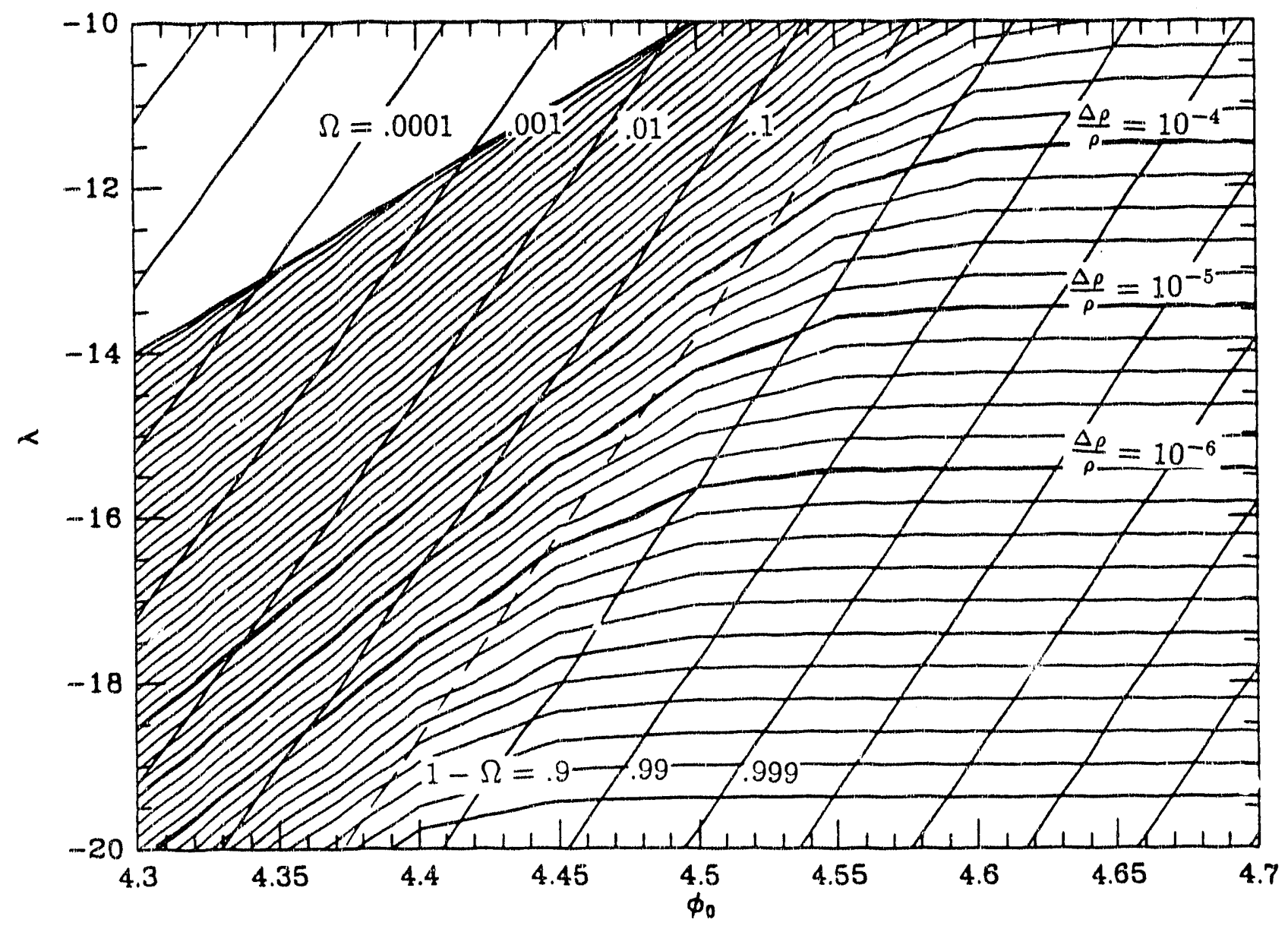

Figure 1. Contours of constant $\Omega$ and $\frac{\Delta \rho}{\rho}$ for different initial values of the potential strength, $\lambda$, and initial inflaton field value, $\phi_{0}$, in a chaotic inflation model with $V(\phi)=$ $\frac{\lambda}{4} \phi^{4}$. Values of $\Omega$ and $(1-\Omega)$ are indicated on the diagonal lines. Contours in constant $\frac{\Delta \rho}{\rho}$ are labeled on the right. 
Thus, the omega problem and flatness problem can be simultaneously satisfied in chaotic inflation (although only at the expense of a "smallness" problem for $\lambda$ ). It is intriguing, therefore, 10 ask what is the probability that the initial value for the inflaton field just happened to be that which would produce a present value of $\Omega \sim 0.1-0.5$.

For the chaotic inflation scenario to be describable by the classical evolution equations we require that $V(\phi) \lesssim M_{P l}^{4}$. 'This limit implies an upper limit to the initial inflaton field amplitude. For example, $\lambda \sim 10^{-12}$, implies $\phi_{0} \lesssim 1000 M_{P l}$. Figure 2 shows the differential probability for a given value of $\Omega$ today under the assumption that any value of $\phi_{0}$ from 0 to 1000 is equally probable. An integral of the region between $0.1 \lesssim \Omega \lesssim 0.5$ gives a probability $\sim 3 \times 10^{-5}$. This integrated probability is not altogether satisfactory. Although it is not vanishingly small, neither is it near unity. One would like to find a reason why our universe might have prefered such a small value for the initial inflaton field. One possibility is that (if the notion of thermal equilibrium is relevant near the Planck time) a Boltzmann factor, or a temperature-dependent, $\mathrm{m}^{2} \mathrm{~T}^{2}$, term in the effective potential might have skewed the probability toward smaller $\phi$. The onset of inflation would quickly decrease the temperature to zero. Nevertheless, the memory of the early thermodynamic history might have favored a small value for $\phi_{0}$. As another possible explanation we consider the stochastic inflation models.

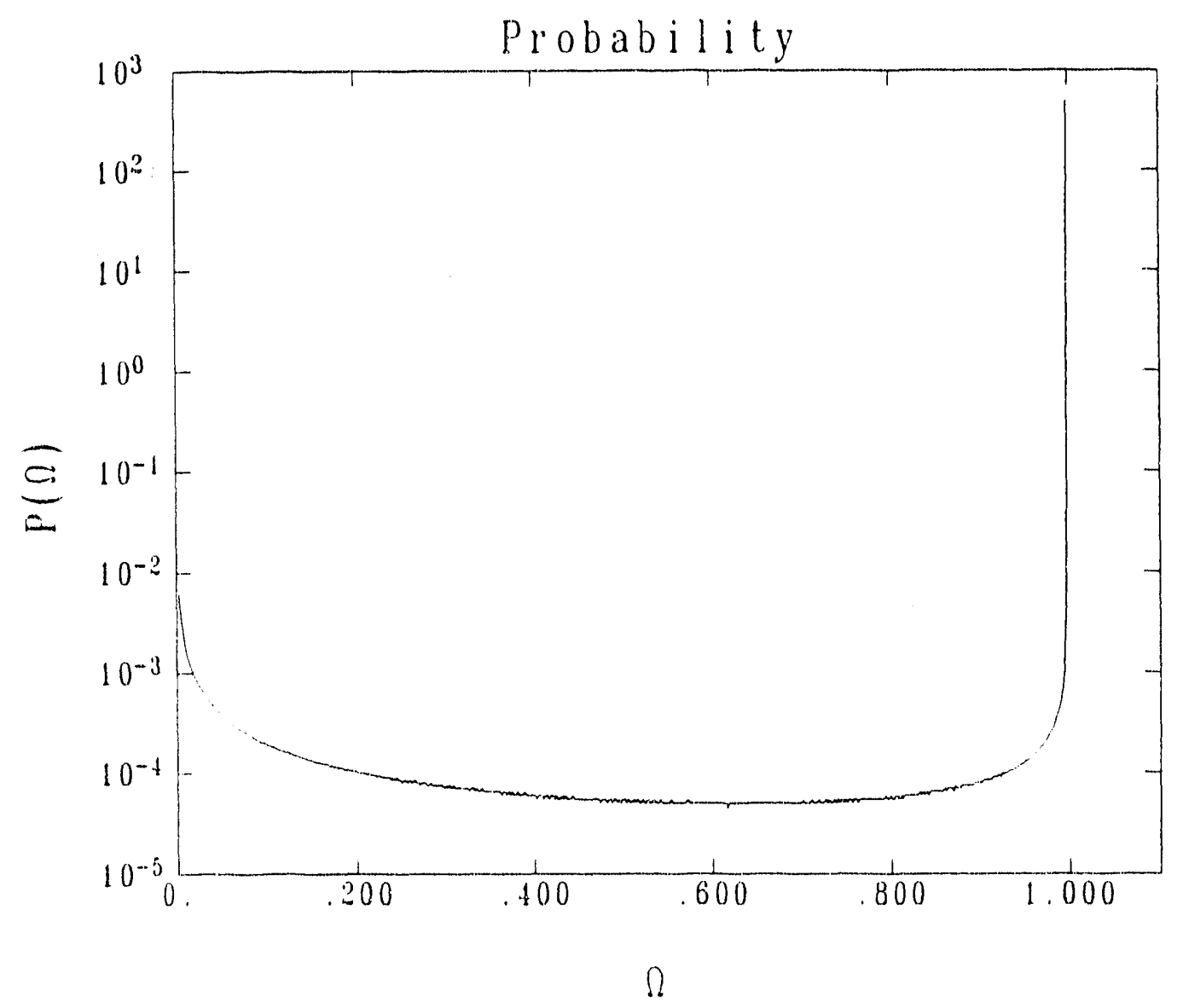

Figure 2. Differential probability for a particular present value of $\Omega$ for a chaotic inflationary model with equal probability for any initial value of the inflaton field from 0 to 1000 $M_{P l}$. 


\section{Stochastic Inflation}

In stochastic inflation (Starobinsky 1984; Graziani and Olynyk 1985; Mazenko 1986; Rey 1987; Goncharov, Linde, and Mukhanov 1987; Bardeen and Bublik 1987; Graziani 1.988; Ortolan, Lucchin, and Matarrese 1988; Nambu, and Sasaki 1989) one assumes the existence of semiclassical long range quantum fluctuations in the inflaton field such that inflation occurs in an ensemble of subdomains each of which creates new subdomains as it inflates. The fact that there is at least some probability which can random walk up the potential, and the fact that higher field values are associated with larger inflation, together imply that the inflation process never ends, i.e. there is a continuous creation of new inflating subdomains (Starobinsky 1984). As Starobinsky (1984) and others (cf. Goncharov, Linde and Mukhanov; Nambu and Sasaki 1989) have shown, the slow-rolling stochastic evolution can be described in terms of a Fokker-Planck equation,

$$
\frac{d P(\phi)}{d t}=\frac{\partial}{\partial \phi}[K P(\phi)]+\frac{\partial^{2}}{\partial \phi^{2}}[D P(\phi)]
$$

where the diffusion coefficients in the slow rolling de Sitter phase are given by,

$$
K \approx \frac{V^{\prime}}{3 H}
$$

and

$$
D \approx \frac{H^{3}}{8 \pi^{2}}
$$

The long-time solution of this equation (Starobinsky 1984) then gives the desired prob-ability distribution for different values of the inflaton field before classical roll over and thermalization.

$$
P(\phi) \sim \exp \left[\frac{3 M_{P l}^{4}}{8}\left(\frac{1}{V(\phi)}-\frac{1}{V\left(\phi_{0}\right)}\right)\right] .
$$

Since this solution assumes slow rolling and only de Sitter evolution, it is only valid for $1 \lesssim \phi \lesssim 1000$. Nevertheless, it has the desired property of maximum probability for small $\phi$. Unfortunately, however, it is too good. For $\lambda \sim 10^{-12}$, the probability is vanishingly small for any values of $\phi$ greater than unity (including the desired value of $\phi \sim 4.5$ ). Our idea has been to look at this distribution more carefully to see if the singularity near small $\phi$ can be avoided. Our reasoning is as follows. The physical probability distribution will be given by the field probability distribution in eq. (5.4) times a factor accounting for the infla'ed volume of the domain (Goncharov, Linde, and Mukharov 1987).

$$
P_{p}=P(\phi) e^{3} \int H d t
$$

The volume factor is a steeply increasing function, $\phi \sim e^{\phi^{2}}$. Thus, if a way can be found to disperse the singularity near $\phi=0$ such that the probability distribution appears gaussian, $P(\phi) \sim e^{\frac{-\phi^{2}}{\sigma^{2}}}$, then the physical probability distribution will have a peak at $\phi \sim 4.5$ for $\sigma$ sufficiently large, $\sigma \sim 5 M_{P l}$. 
Our approach has been to study the evolution of a more general Fokker.-Planck equation which is valid through the transition to a Friedmann expansion and which does not rely upon the slow-rolling approximation which breaks down near the bottom of the potential. We do, however, neglect gravitational back reaction-effects. The present work should thus be thought of as a mean field approximation to the exact relativistic solution.

For example, our generalized Fokker-Planck equation for an effective potential of the form $V(\phi)=\frac{m^{2}}{2} \phi^{2}$ is,

$$
\begin{gathered}
\frac{d P(\phi)}{d t}=-\frac{\partial}{\partial \phi}[\xi P]+\frac{\partial}{\partial \xi}[3 H \xi P]+\frac{\partial}{\partial \xi}\left[m^{2} \phi P\right] \\
+\frac{\partial^{2}}{\partial \phi^{2}}\left[D_{\phi \phi} P\right]+\frac{\partial^{2}}{\partial \phi \partial \xi}\left[\left(D_{\phi \xi}+D_{\xi \phi}\right) P\right]+\frac{\partial^{2}}{\partial \xi^{2}}\left[D_{\xi \xi} P\right]
\end{gathered}
$$

where $\xi$ is the conjugate momentum to $\phi$. The diffusion constants are defined in terms of the scale factor and Fourier amplitudes, $\psi_{k}(t)$, for the short-range fluctuating component of $\phi$,

$$
\begin{gathered}
D_{\phi \phi}=\frac{\epsilon^{3}}{4 \pi^{2}}|\ddot{R}| \dot{R}^{2}\left|\psi_{k}(t)\right|^{2} \\
D_{\xi \xi}=\frac{\epsilon^{3}}{4 \pi^{2}}|\ddot{R}| \dot{R}^{2}\left|\dot{\psi}_{k}(t)\right|^{2} \\
D_{\phi \xi}+D_{\xi \phi}=\frac{\epsilon^{3}}{4 \pi^{2}}|\ddot{R}| \dot{R}^{2} \frac{\partial}{\partial t}\left|\psi_{k}(t)\right|^{2} .
\end{gathered}
$$

The diffusion coefficients are evaluated at $k=\epsilon \dot{R}$ where $\epsilon$ is a small parameter, $\epsilon \ll 1$.

Our hope has been that by studying a more general Fokkcr-Planck solution the singularity in the long term solution can be avoided. Our studies of the behavior of this more general equation, however, have indicated that the quantum fluctuations damp out significantly by the time the distribution populates the bottom of the po tial. Figure 3 demonstrates how neither classical oscillations of the field nor thermal fluctuations during reheating could produce a large enough dispersion in the long time solution to produce the desired peak.

Figure 3 shows the envelope of trajectories of the classical field at the bottom of the potential for different initial values of the inflaton field. This calculation is in the limit of weak reheating. Note that even without reheating the maximum amplitude of the oscillations is only $\sim 0.1 \mathrm{MPl}$. Thus, classical oscillations of the field do not provide enough width to the distribution. Similarly, in the limit of strong reheating, the amount of thermal energy available is miniscule compared to the amount of vacuurn energy required to produce a significant fattening of the distribution. So thermal fluctuations do not solve the problem either. 


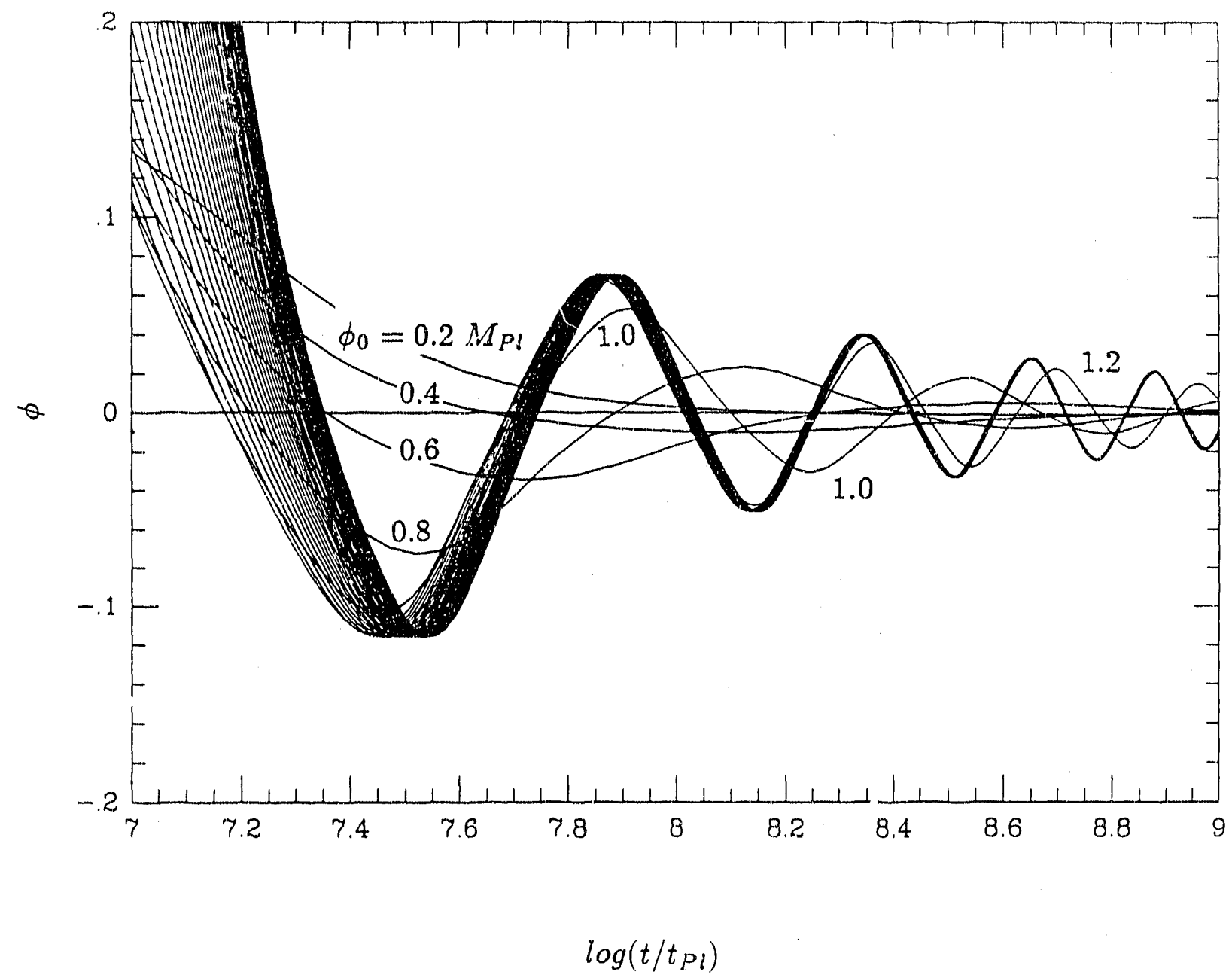

Figure 9. Envelope of the time evolution of the inflaton field starting from different initial values, $\phi_{0}$.

We note that one possible solution to this problem is at least hinted at in the recent work of Nambu and Sasaki (1989). They showed that by explicitly including the volume term into the Fokker-Planck equation, i.e.

$$
\frac{d P(\phi)}{d t}=\frac{\partial}{\partial \phi}[K P(\phi)]+\frac{\partial^{2}}{\partial \phi^{2}}[D P(\phi)]+3(H-<H>) P(\phi),
$$

that an analytical solution can be found which avoids the singularity for small $\phi$. Indeed their solution ever appears to allow for a peak in the disiribution for finite $\phi$. Figure 4 shows examples of the analytic solution from Namou ans Sasaki (1989) for several different values of $\lambda$. It appears possible to have a peak in the cistribution for $\phi \sim 5$. However, for this value of $\lambda$ the microwave background fluctuations are unacceptably large, ard the peak occurs at a value for the potential energy which is greater than the Planck scale.

The only way we have found to solve the $\Omega$ problem in the context of stochastic inflation is by utilizing a generalized effective potential of the form,

$$
V(\phi)=\lambda\left[\frac{\alpha}{2} \phi^{2}+\frac{\beta}{3} \phi^{3}+\frac{1}{4} \phi^{4}\right] .
$$


The distribution shown in figure 5 was generated from such a potential with $\alpha=2.32$, $\beta=-3.02$, and $\lambda=10^{-12}$. A universe which rolls down from the peak in the distribution would have present fluctuations in the microwave background of $\frac{\Delta T}{T} \sim 5 \times 10^{-5}$ and $\Omega \sim 0.3$.

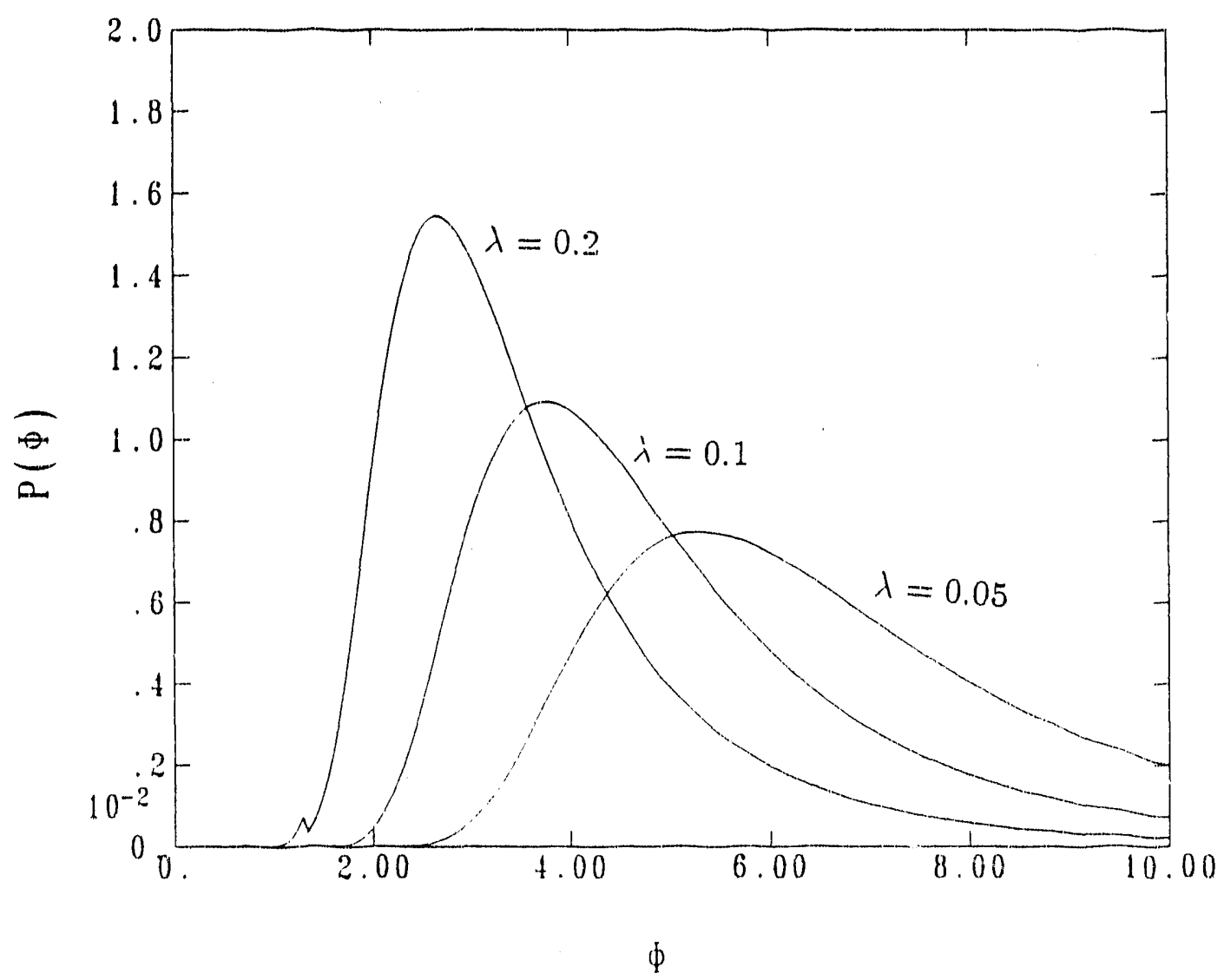

Figure 4. Stationary renormalizable analytic solution (Nambu and Sasali 1989) for the probability distribution of $\phi$ for different potential strengths, $\lambda$. 


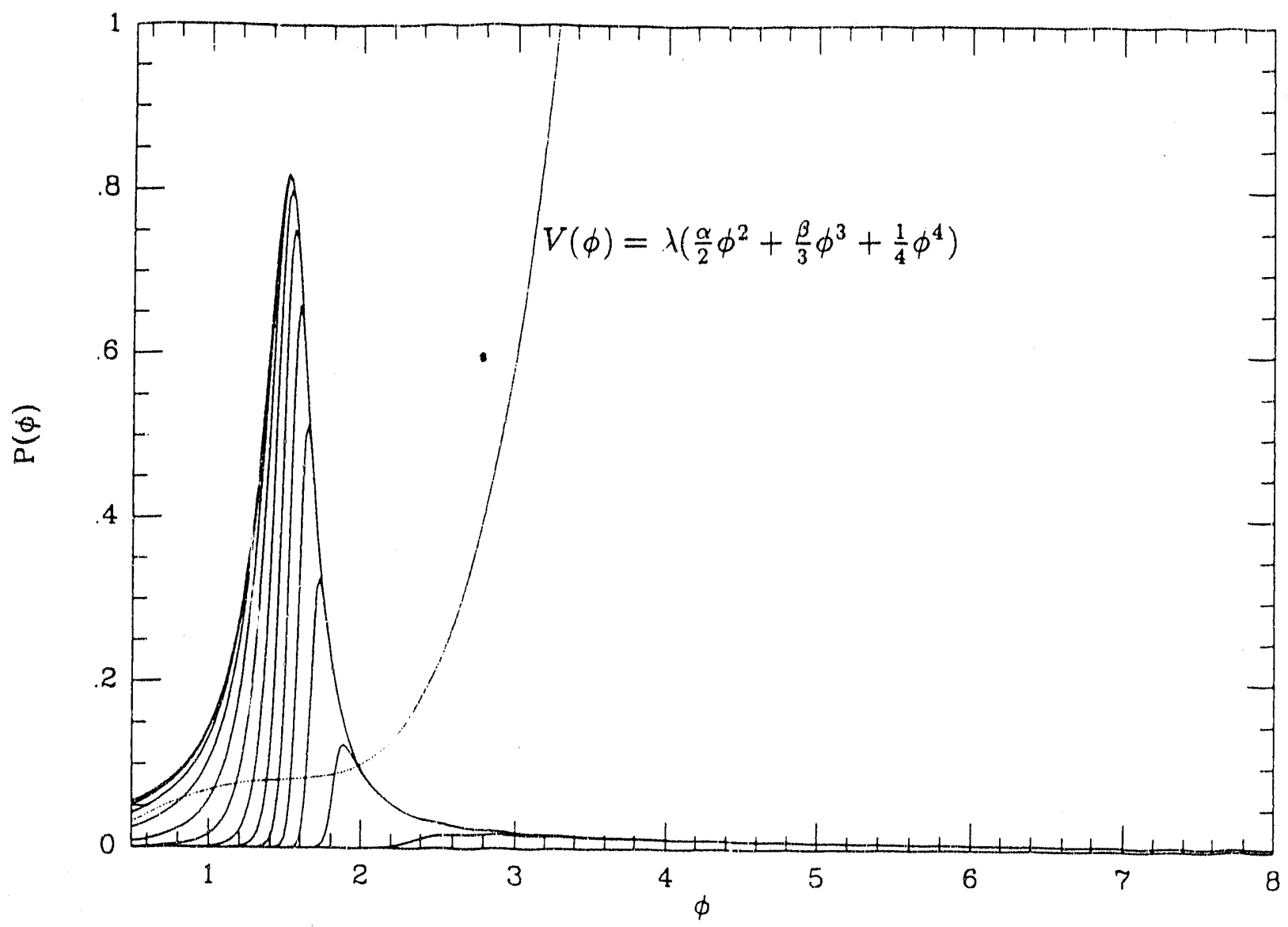

Figure 5. Example of the development of a stationary solution for $P(\phi)$ from the stochastic evolution of a system with a generalized effective potential. For this calculation probability flows in from the right as new universes are born and exits on the left as universes roll down the potential and thermalize.

The authors acknowledge iseful conversations with N. J. inyderman, M. J. Rees, and J. R. Bond. Work performed under the auspices of the U. S. Department of Energy by the Lawrence Livermore National Laboratory under contract W-7405-ENG-48.

\section{REFERENCES}

Audouze, J. 1990 (this Conf. Proc.).

Bahcall, S. R. and Tremaine, S. 1988, Ap. J. Lett., 326, L1

Bardeen, J. and Bublik, G. J. 1987, Class. Quant Grav., 4, 573.

Caditz, D. and Petrosian, V. 1989, Ap. J. Lett., 337, L65.

Davis, M. and Peebles, P. J. E. 1983, Ann. Rev. Astr. Ap., 21, 109.

Fukugita, M. 1990 (this Conf. Proc.); and "Proc. 3rd Nishinomiya Yukawa Mernorial Symposium," Nishinomiya, Japan. 10-11 Nov. 1988.

Fukugita, M., Takahara, F., Yamashita. K., and Yoshii, Y. 1990, Subritted to Ap. J. 
Goncharov, A. S., Linde, A. D., and Mukhanov, V. F. 1987, Int. J. Mod. Phys., A2, 561. Graziani, F. 1988, Phys. Rev., D38, J122, 1131, 1808.

Ia, D. and Steinhardt. P. J. 1989, Phys. Rev. Lett., 62, 376.

Linde, A. D. 1983, Phys. Lett., B129 177.

Loh, E and Spillar, E. J. 1986, Ap. J. Lett., 307, L1.

Mathews, G. J., Meyer, B. S., Alcock, C. R., and Fuller, G. M. 1990, Ap. J., 358, 36.

Mazenko, C. 1986, Fins. Rev., D 34, 2223.

Narnbu, Y. and Sasaki, M. 1989, Phys. Lett., B219, 240.

Ortolan, A., Lucchin, F., and Matarrese, S. 1988, Phys. Rev, D 38, 465.

Feebles, P. J. E. 1975, Ap. J., 205, 318.

Rey, S. J. 1987, Nucl. Phys., B284, 706.

Sardage, A. 1938, Ann. Rev. Astron. Ap, 26, 561.

Sato, K. 1990 (ihis Conf. Proc.).

Starobinsky, A. A. 1984, in Fundamental Interactions, V. N. Ponomarev, ed., (MPGI press, Moskcin) p. 54.

Steigmar, G. 1390 (this Conf. Proc.).

Steinh.srdt P. J. 1990, Nature, 345, 47.

Yahil, A., Walker, D., and Rowan-Robinson, M. 1986, Ap. J. Lett., 301, L1.

Yoshii, Y. and Takahara, F. 1988, Ap. J., 326, 1. 

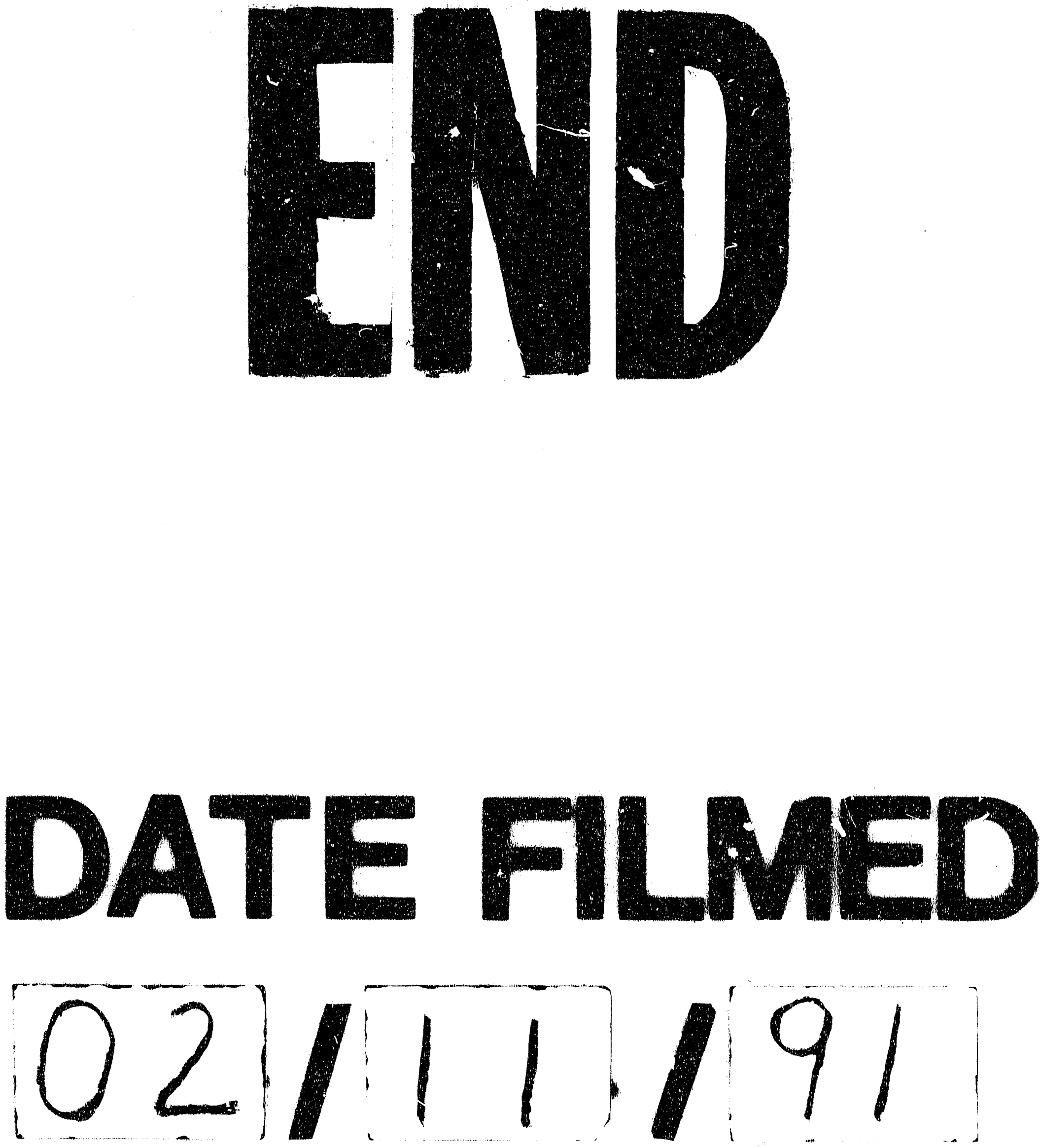
